\title{
O papel do enfermeiro na busca ativa de pacientes em abandono do tratamento de tuberculose: uma revisão integrativa da literatura
}

\author{
The role of nurses in the active search for patients abandoning tuberculosis treatment: an \\ integrative literature review \\ El papel de las enfermeras en la búsqueda activa de pacientes que abandonan el \\ tratamiento de la tuberculosis: una revisión integral de la literatura
}

Denise Silva Araújo ${ }^{1 *}$, Marilene de Sousa Palheta ${ }^{1}$, Janaína Cosarde de Souza ${ }^{1}$, Ivandir de Carvalho Passos Júnior ${ }^{1}$, Francinilson Bernardo da Silva ${ }^{1}$, Vanessa de Souza Correia de Araújo ${ }^{1}$, Rhuana Maria de Oliveira Pereira ${ }^{2}$, Jéssica Karoline Alves Portugal ${ }^{2}$, Marcelo Henrique da Silva Reis ${ }^{2}$, Lucas da Silva de Almeida².

\section{RESUMO}

Objetivo: Descrever através de uma revisão sobre o papel do profissional enfermeiro na busca ativa de pacientes no abandono do tratamento de tuberculose pulmonar. Métodos: Trata-se de uma Revisão Integrativa de Literatura que pretendeu descrever o papel do profissional enfermeiro na busca ativa de pacientes no abandono do tratamento de tuberculose. As bases de dados para a coleta de dados foram: SCIELO, LILACS, MEDLINE e BDENF. Os critérios de inclusão foram: textos publicados nos últimos 10 anos, artigos originais, publicados em texto completo, publicados em língua portuguesa, disponibilizados gratuitamente. Resultados: Para elaboração desta revisão de literatura foram identificados na base de dados um total de 5 artigos. Os tipos de estudo da amostra em sua maioria eram descritivos, e têm por objetivo determinar a distribuição de doenças ou condições relacionadas à saúde. A abordagem quantitativa corresponde a $80 \%$ da amostra, essa abordagem é uma classificação do método científico que utiliza diferentes técnicas estatísticas para quantificar opiniões e informações para um determinado estudo. Considerações finais: $O$ enfermeiro exerce um papel fundamental na busca ativa de pacientes que estejam abandonando o tratamento de tuberculose, através da elaboração de estratégias de acompanhamento satisfatórias, favorecendo a adesão ao tratamento, executando políticas públicas já existentes.

Palavras-chave: Tuberculose, Atenção primária à saúde, Pacientes desistentes do tratamento.

\section{ABSTRACT}

Objective: To Describe through a review of the role of the professional nurse in the active search for patients in the abandonment of pulmonary tuberculosis treatment. Methods: This is an Integrative Literature Review that aimed to describe the role of the professional nurse in the active search for patients in the abandonment of tuberculosis treatment. The databases for data collection were: SCIELO, LILACS, MEDLINE and BDENF. The inclusion criteria were: texts published in the last 10 years, original articles, published in full text, published in Portuguese, available for free. Results: In order to prepare this literature review, a total of 5 articles were identified in the database. The types of study in the sample were mostly descriptive, and aim to determine the distribution of diseases or conditions related to health. The quantitative approach corresponds to $80 \%$ of the sample, this approach is a classification of the scientific method that uses different statistical techniques to quantify opinions and information for a given study. Final considerations: Nurses play a fundamental role in the active search for patients who are abandoning tuberculosis treatment, through the development of satisfactory follow-up strategies, favoring adherence to treatment, implementing existing public policies.

Keywords: Tuberculosis, Primary health care, Patients dropping out of treatment.

\section{RESUMEN}

Objetivo: Describir a través de una revisión del papel de la enfermera profesional en la búsqueda activa de pacientes en el abandono del tratamiento de la tuberculosis pulmonar. Métodos: Esta es una revisión de literatura integradora que tuvo como objetivo describir el papel del profesional de enfermería en la búsqueda activa de pacientes en el abandono del tratamiento de la tuberculosis. Las bases de datos para la recolección

\footnotetext{
${ }^{1}$ Centro Universitário do Norte (UNINORTE), Manaus - AM. *E-mail: reis.henrique.marcelo@gmail.com

2 Universidade Federal do Amazonas (UFAM), Coari - AM.
}

SUBMETIDO EM: 6/2020 
de datos fueron: SCIELO, LILACS, MEDLINE y BDENF. Los criterios de inclusión fueron: textos publicados en los últimos 10 años, artículos originales, publicados en texto completo, publicados en portugués, disponibles de forma gratuita. Resultados: Para preparar esta revisión de la literatura, se identificaron un total de 5 artículos en la base de datos. Los tipos de estudio en la muestra fueron en su mayoría descriptivos y tienen como objetivo determinar la distribución de enfermedades o afecciones relacionadas con la salud. El enfoque cuantitativo corresponde al $80 \%$ de la muestra, este enfoque es una clasificación del método científico que utiliza diferentes técnicas estadísticas para cuantificar opiniones e información para un estudio dado. Consideraciones finales: Las enfermeras desempeñan un papel fundamental en la búsqueda activa de pacientes que abandonan el tratamiento de la tuberculosis, mediante el desarrollo de estrategias de seguimiento satisfactorias, que favorecen la adherencia al tratamiento y la implementación de las políticas públicas existentes.

Palabras clave: Tuberculosis, Atención primaria de salud, Pacientes que abandonan el tratamiento.

\section{INTRODUÇÃO}

A Tuberculose (TB) é delineada como um agravamento infectocontagioso que vem sendo descrita ao longo da história e que persiste como um problema de saúde pública preocupante, a mesma origina-se através do bacilo aeróbico Mycobacterium tuberculosis ou Bacilo de Koch (BK) (ALENCAR IFPS, et al., 2019). Esta patologia sugere preferência por um órgão específico, sendo este os pulmões, porém o bacilo pode alvejar outros órgãos como: os ossos, rins, meninges, gânglios e outros. A tuberculose é uma enfermidade que se apresenta com uma particularidade marcante que é seu longo período de latência entre a infecção inicial até a as suas manifestações clínicas (ALENCAR IFPS, et al., 2019).

A tuberculose pulmonar (TBP) evidencia sinais clínicos que podem ser confundidos com a gripe, os mais comuns são "inapetência, febre vespertina, rouquidão, sudorese noturna, fadiga, perda ponderal e o sinal de extrema relevância que é a tosse contínua por semanas", esses aspectos acabam dificultando o diagnóstico imediato da tuberculose. O diagnóstico da TBP é baseado na história clínica e epidemiológica, baciloscopia e radiografia e cultura de escarro na maioria dos casos (LIMA EA, et al., 2019).

A transmissão acontece por via aérea, o indivíduo que está contaminado pela tuberculose dissemina o bacilo para outro saudável, através das gotículas de salivas expelidas durante a fala, risos, espirro, tosse e respiração. O declínio da imunidade e o aglomerado de pessoas em determinada localidade estão associadas as formas de transmissibilidade (LIMA EA, et al., 2019).

A maior incidência da TB localiza-se na periferias de grandes cidades, visto que é significativa a concentração populacional, tornando-a prevalente em regiões com os serviços de infraestrutura reduzidos e saneamento básico insalubre, outra localidade onde há altas taxas evidentes da doença são moradas que possuem ambientes fechados, escuro e com pouquíssima ventilação, exemplificando assim ambientes prisionais, logo, pode-se mencionar que esta doença está diretamente associada, às condições de vida precárias persistindo como um grave problema de saúde pública, contribuindo para o quadro de desigualdade e exclusão social (BRASIL, 2018).

"No mundo, em 2018, cerca de dez milhões de pessoas adoeceram por tuberculose e 1,5 milhão de pessoas morreram em decorrência dela, sendo a TB a principal causa de morte por um único agente infeccioso, o que mantém a TB entre as 10 principais causas de morte no planeta, no Brasil, em 2019, 487 casos de TB foram notificados após o óbito" (BRASIL, 2020).

O Programa Nacional de Controle da Tuberculose (PNCT) é agregado na rede de serviços de saúde, é desenvolvido por meio de um programa unificado e executado pelas esferas federativa, estatal e municipal. A política é estabelecida por um conjunto de ações com padrões técnicos e assistenciais, garantindo desde a oferta de medicamentos e insumos gratuitos até ações e medidas estratégicas para prevenção e controle do agravo, permitindo o acesso universal da população (BRASIL, 1999).

A equipe que auxilia desde o acolhimento, diagnóstico e tratamento é composta por multiprofissionais, dentro da detecção inicial da doença o PNCT orienta que o indivíduo dê entrada através da atenção primária. Diante disto, o profissional enfermeiro tem papel essencial no controle da TB, realizando orientações à população referente à doença, esclarecendo as formas de transmissão e principalmente enfatizando a 
importância da adesão ao tratamento completo e adequado. Entende-se adesão como um processo dinâmico e multifatorial, influenciada por fatores individuais e externos, para que haja adesão ao tratamento um dos principais determinantes é o vínculo do paciente-profissional (FRAGA IMN, et al., 2019).

$\mathrm{O}$ abandono ao tratamento acarreta ao indivíduo potencialidade para se tornarem portadores de bacilos multirresistentes, desta forma o profissional enfermeiro é responsável por realizar a busca ativa dos pacientes desistentes do tratamento. Para o sucesso do tratamento, deve-se contar com o comprometimento de toda a Estratégia Saúde da Família (ESF) no desenvolvimento das atividades, além da responsabilização pela saúde da comunidade. O principal objetivo da busca ativa é assegurar a realização completa e eficaz do tratamento para evitar assim a transmissibilidade e proliferação descontrolada da TB (FERREIRA MRL, et al., 2019).

Perante o exposto, surge algumas inquietações referente de como ocorre a busca ativa de pacientes desistente do tratamento de TB abordando com isso a seguinte pergunta norteadora: qual o papel do profissional enfermeiro na busca ativa de pacientes no abandono do tratamento de tuberculose pulmonar na atenção primária?

Neste contexto, o objetivo deste trabalho é descrever, através de uma revisão integrativa da literatura, qual o papel do profissional enfermeiro na busca ativa de pacientes no abandono do tratamento de tuberculose pulmonar na atenção primária.

\section{MÉTODOS}

Trata-se de um estudo de Revisão Integrativa da Literatura (RIL), realizado a partir de pesquisas em artigos científicos publicados nas bases de dados Scientific Electronic Library Online (SCIELO), Literatura LatinoAmericana em Ciências da Saúde (LILACS), Literatura Internacional em Ciências da Saúde (MEDLINE) e Base de dados em Enfermagem (BDENF). A revisão integrativa da literatura é um método que determina o conhecimento específico sobre uma temática, visando identificar, analisar e sintetizar resultados de estudos independentes que retratam o mesmo assunto, para uma possível repercussão favorável na qualidade dos cuidados prestados ao paciente.

Como critérios de inclusão foram estabelecidos artigos em texto completo, em idioma português, publicados no intervalo de janeiro de 2010 a janeiro de 2020 e que correspondessem aos objetivos da revisão. Os critérios de exclusão foram: estudos do tipo teses, dissertações, monografias, revisão de literatura e textos publicados em língua estrangeira. Por tratar-se de um estudo de revisão da literatura, não foi necessária a submissão do trabalho no Comitê de Ética em Pesquisa (CEP), no entanto, a elaboração foi realizada respeitando as normas de citação e preservação dos direitos autorais vigentes.

\section{RESULTADOS}

Para elaboração desta revisão de literatura foram identificados na base de dados um total de 5 artigos. Na base SCIELO foram encontradas inicialmente 1 referência, após a aplicabilidade dos filtros baseados nos critérios de inclusão e exclusão e leitura minuciosa, foi selecionado 1 artigo para compor a amostra. Na base de dados LILACS, foram obtidas 16 publicações, após a filtragem utilizando os critérios de inclusão e exclusão, restaram 4 artigos, após a leitura minuciosa restaram 3 artigos para compor a amostra. Na base de dados MEDLINE foram obtidas 2 publicações, após a filtragem utilizando os critérios de inclusão e exclusão, o resultado de pesquisa foi 0 , não havendo publicações para compor a amostra.

$\mathrm{Na}$ base de dados BDENF foram levantadas 2 publicações, após a aplicabilidade dos filtros baseados no critério de inclusão e exclusão e leitura minuciosa foi selecionado 1 artigos para a composição da amostra. Assim, a amostra final utilizada para compor esta pesquisa foi de 5 artigos conforme evidenciado na Figura 1.

As avaliações das variáveis Base, Revista, Titulo, Autores, Objetivo, Tipo de estudo, Abordagem e Ano demonstraram que, na base de dados SCIELO foi selecionado 1 artigo, na base de dados LILACS foi selecionado 3 artigos, na base de dados MEDLINE não foi encontrado nenhum artigo para compor a amostra, na base de dados BDENF foram selecionados 1 artigos, resultando no total de 5 artigos para a composição da revisão de literatura. Foram encontrados os autores conforme estão descritos no Quadro 1. 
Figura 1 - Fluxograma metodológico para seleção de artigos.

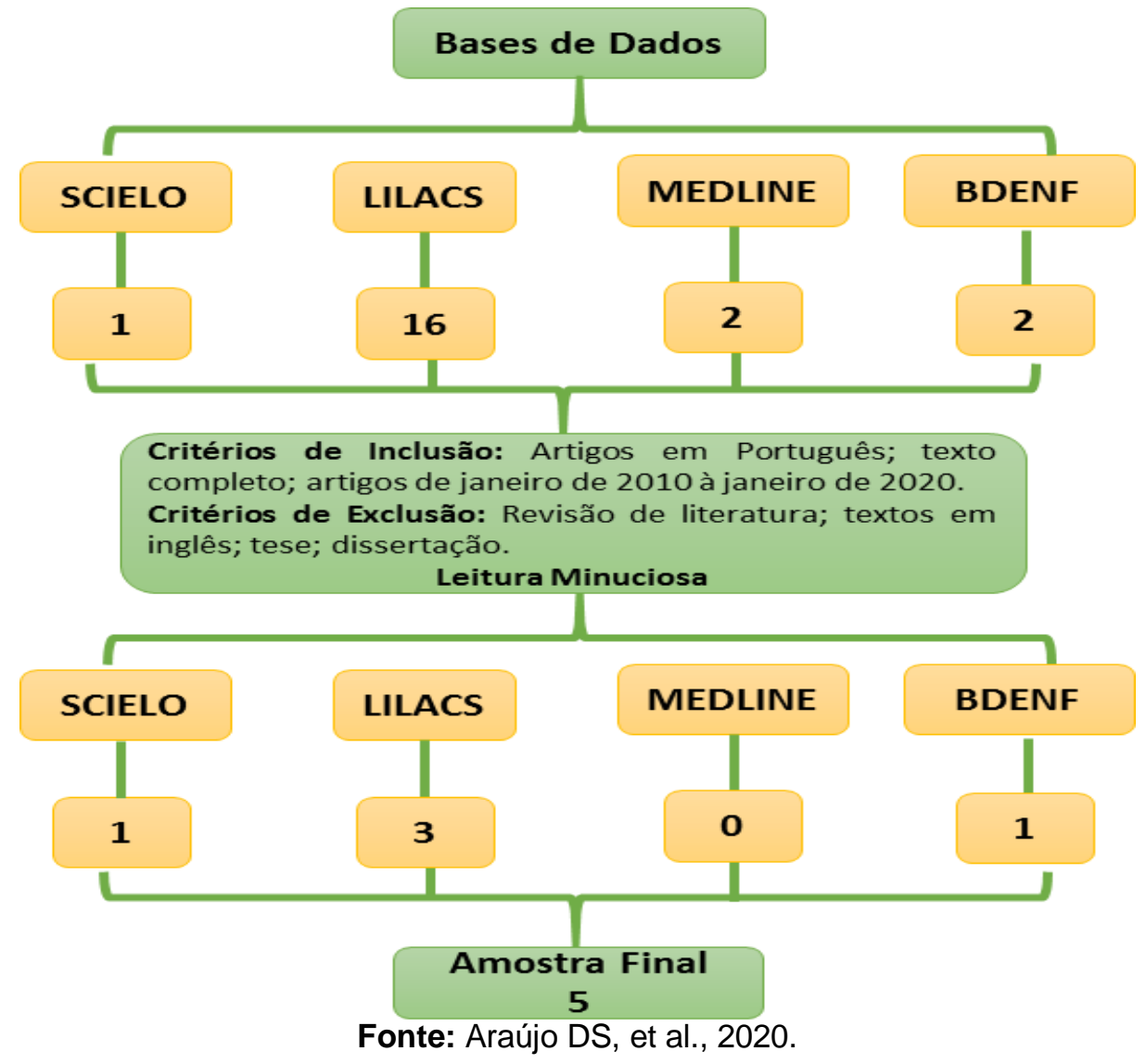

Analisando o Quadro 1 observamos que os artigos trazem como título, respectivamente: "Fatores preditores para o abandono do tratamento da tuberculose pulmonar preconizado pelo Ministério da Saúde do Brasil na cidade de Porto Alegre (RS)", "Avaliação do acompanhamento e desfecho de casos de tuberculose em município do sul do Brasil", "Efetividade do tratamento da tuberculose", "Fatores associados ao abandono do tratamento da tuberculose nos serviços de atenção básica em dois municípios brasileiros, Manaus e Fortaleza, 2006 a 2008" e "Razões que levam os pacientes com tuberculose a abandonarem o tratamento: perspectivas do enfermeiro".

As revistas selecionadas a partir das bases de dados foram: Jornal Brasileiro de Pneumologia - que destina-se a publicar artigos científicos que contribuam para o aumento do conhecimento no campo das doenças pulmonares e áreas relacionadas. A Revista Gaúcha de Enfermagem - da Universidade Federal do Rio Grande do Sul, que busca contribuir para a disseminação do conhecimento em saúde, publicando a produção científica na área de Enfermagem.

O Caderno de Saúde Coletiva, do Instituto de Estudos em Saúde Coletiva da Universidade Federal do Rio de Janeiro que objetiva contribuir com a consolidação e o desenvolvimento do campo do conhecimento em Saúde Coletiva, afirmando seu caráter multidisciplinar e seu compromisso com as transformações sociais, que resultam em benefícios para a saúde das populações humanas, através da divulgação de estudos originais teóricos e / ou aplicados que são interessantes para a saúde pública. E a Revista Enfermagem Atual - é uma revista científica da Sociedade Brasileira de Enfermagem em Feridas e Estética (SOBENFeE), publicada trimestralmente, voltada para o público de profissionais e acadêmicos e pós-graduados da área de saúde.

Os tipos de estudo da amostra em sua maioria eram descritivos, e têm por objetivo determinar a distribuição de doenças ou condições relacionadas à saúde, segundo o tempo, o lugar e/ou as características dos indivíduos (FACURI CO, et al., 2013). A abordagem quantitativa corresponde a $80 \%$ da amostra, essa abordagem é uma classificação do método científico que utiliza diferentes técnicas estatísticas para quantificar opiniões e informações para um determinado estudo (SOUSA LO, et al., 2016). 
Quadro 1 - Caracterização dos estudos selecionados, segundo as bases de dados: Base, Revista, Título, Autores, Objetivo e Metodologia e Ano.

\begin{tabular}{|c|c|c|c|c|c|c|c|c|}
\hline \multirow[b]{2}{*}{ № } & \multirow[b]{2}{*}{ Base } & \multirow[b]{2}{*}{ Revista } & \multirow[b]{2}{*}{ Título } & \multirow[b]{2}{*}{ Autor (es) } & \multirow[b]{2}{*}{ Objetivo } & \multicolumn{2}{|c|}{ Metodologia } & \multirow[b]{2}{*}{ Ano } \\
\hline & & & & & & $\begin{array}{l}\text { Tipo de } \\
\text { estudo }\end{array}$ & Abordagem & \\
\hline 1 & SCIELO & $\begin{array}{l}\text { Jornal Brasileiro } \\
\text { de Pneumologia }\end{array}$ & $\begin{array}{l}\text { Fatores preditores para o } \\
\text { abandono do tratamento } \\
\text { da tuberculose pulmonar } \\
\text { preconizado pelo } \\
\text { Ministério da Saúde do } \\
\text { Brasil na cidade de Porto } \\
\text { Alegre (RS) }\end{array}$ & $\begin{array}{l}\text { CAMPANI } \\
\text { STA, et al. }\end{array}$ & $\begin{array}{c}\text { Determinar os fatores preditores de } \\
\text { abandono do tratamento da tuberculose } \\
\text { pulmonar, preconizado pelo Ministério da } \\
\text { Saúde do Brasil, em pacientes bacilíferos } \\
\text { virgens de tratamento atendidos na cidade } \\
\text { de Porto Alegre (RS). }\end{array}$ & Caso-controle & Quantitativo & 2011 \\
\hline 2 & LILACS & $\begin{array}{l}\text { Revista Gaúcha } \\
\text { de Enfermagem }\end{array}$ & $\begin{array}{l}\text { Avaliação do } \\
\text { acompanhamento e } \\
\text { desfecho de casos de } \\
\text { tuberculose em município } \\
\text { do sul do Brasil }\end{array}$ & $\begin{array}{l}\text { HARTER J, et } \\
\text { al. }\end{array}$ & $\begin{array}{l}\text { Avaliar o acompanhamento e desfecho do } \\
\text { tratamento de casos de tuberculose } \\
\text { pulmonar no programa de controle da } \\
\text { tuberculose de um município prioritário do } \\
\text { Sul do Brasil. }\end{array}$ & Descritivo & Quantitativo & 2016 \\
\hline 3 & LILACS & $\begin{array}{l}\text { Jornal Brasileiro } \\
\text { de Pneumologia }\end{array}$ & $\begin{array}{l}\text { Efetividade do tratamento } \\
\text { da tuberculose }\end{array}$ & $\begin{array}{l}\text { PAZ LNF, et } \\
\text { al. }\end{array}$ & $\begin{array}{l}\text { Analisar as estratégias que influenciam a } \\
\text { efetividade do tratamento da tuberculose em } \\
\text { unidades Básicas de Saúde (UBS). }\end{array}$ & Descritivo & Quantitativo & 2012 \\
\hline 4 & LILACS & $\begin{array}{l}\text { Caderno de } \\
\text { Saúde Coletiva }\end{array}$ & $\begin{array}{c}\text { Fatores associados ao } \\
\text { abandono do tratamento } \\
\text { da tuberculose nos } \\
\text { serviços de atenção } \\
\text { básica em dois } \\
\text { municípios brasileiros, } \\
\text { Manaus e Fortaleza, 2006 } \\
\text { a } 2008\end{array}$ & $\begin{array}{l}\text { BRAGA JU, et } \\
\text { al. }\end{array}$ & $\begin{array}{c}\text { Identificar os fatores de risco para o } \\
\text { abandono de tratamento da TB nos cenários } \\
\text { da atenção básica de saúde, dos municípios } \\
\text { de Manaus e Fortaleza e avaliar a influência } \\
\text { da distância entre o local de atendimento e a } \\
\text { residência dos pacientes no abandono ao } \\
\text { tratamento. }\end{array}$ & Caso-controle & Quantitativo & 2012 \\
\hline 5 & BDENF & $\begin{array}{l}\text { Revista } \\
\text { Enfermagem } \\
\quad \text { Atual }\end{array}$ & $\begin{array}{l}\text { Razões que levam os } \\
\text { pacientes com } \\
\text { tuberculose a } \\
\text { abandonarem o } \\
\text { tratamento: perspectivas } \\
\text { do enfermeiro }\end{array}$ & $\begin{array}{l}\text { SACKSER } \\
\text { MA e } \\
\text { BORGES } \\
\text { AM. }\end{array}$ & $\begin{array}{l}\text { Identificar os motivos que levam os pacientes } \\
\text { a desistir do tratamento da tuberculose no } \\
\text { município de Santa Cruz do Sul, a partir da } \\
\text { visão dos enfermeiros que atuam em } \\
\text { unidades de atenção primária de saúde. }\end{array}$ & Descritivo & Qualitativo & 2019 \\
\hline
\end{tabular}

Fonte: Araújo DS, et al., 2020. 


\section{DISCUSSÃO}

Após a análise dos artigos selecionados para constituir a amostra da pesquisa, utilizamos blocos de conteúdo temáticos que conduziram os achados frequentes, como forma de descrever o papel do profissional enfermeiro na busca ativa de pacientes no abandono do tratamento de tuberculose pulmonar na atenção primária.

\section{O papel do enfermeiro na assistência de enfermagem ao paciente com TB}

Campani STA, et al. (2011) (artigo 1), elucida que o papel do enfermeiro perante à tuberculose está vinculado desde o primeiro atendimento, onde deve ser avaliado a história clínica do paciente, realização do exame físico completo e observação dos sinais e sintomas relatados pelo indivíduo. Paz LNF, et al. (2016) (artigo 3), complementa que ao serem observadas manifestações como: sudorese noturna, tosse produtiva, fadiga, perda de peso, anorexia e febre, cabe ao enfermeiro atuar junto ao diagnóstico da doença, avaliando os sinais e sintomas manifestados pelo paciente, após julgamento clínico solicitar exame de Baciloscopia, Bacilos Álcool-Ácido Resistentes (BAAR).

As diretrizes do Programa Nacional de Controle da Tuberculose do Ministério da Saúde, enfatizam a importância de inúmeras condutas que o profissional enfermeiro junto a equipe multidisciplinar, devem realizar para garantir o sucesso no tratamento e o controle da doença, essas condutas devem ser executadas cumprindo primordialmente de acordo com que a Política Nacional de Controle da Tuberculose preconiza (BRASIL, 2018).

As principais condutas exigidas são a realização do acompanhamento e orientação para adesão ao tratamento, orientar a coleta do exame, busca Ativa de Sintomáticos Respiratório, Tratamento Diretamente Observado (TOD), promover a atividade e a nutrição adequada e monitorar as complicações potenciais. Além disso, a evolução, os resultados obtidos com o tratamento, devem ser analisadas e registradas pelo enfermeiro e o caso com seu respectivo desfecho deve ser notificado à secretaria de saúde do município (BRASIL, 2020).

\section{Dificuldades do enfermeiro e do paciente para o enfrentamento, controle e prevenção do abandono do tratamento para TB}

Alguns fatores estão intimamente associados as dificuldades do enfermeiro para o enfrentamento, controle e prevenção do abandono do tratamento de tuberculose, dentre esses fatores estão: o grande número de pacientes para serem atendidos, a falta de recursos humanos e a desorganização do serviço (COUTO DS, et al., 2014).

O enfermeiro atuante na atenção primária executa além do papel assistencial, a demanda administrativa, como preenchimento de formulários, livros de programas disponíveis na unidade de saúde, lança a produção em sistemas, muitas vezes gerencia a própria unidade solicitando materiais, suprimento e lidera toda equipe. Todas essas atividades acabam sobrecarregando a demanda do profissional e restringido ou até mesmo diminuindo o número de atendimentos executados pelo mesmo, que por vezes atrapalha o registro de casos (FREITAS PC, et al., 2020).

De acordo com Sackser MA e Borges AM (2019), relataram que o aumento da demanda de atividades dos enfermeiros na unidade de saúde acarreta em descaso no atendimento e registro de casos de TB. A falta de recursos humanos é a dificuldade mais persistente para o enfrentamento, controle e prevenção do abandono do tratamento de tuberculose, pois acarreta na falta de assistência as necessidades dos pacientes que buscam o serviço, tanto no âmbito multiprofissional quanto no administrativo (SACKSER MA, BORGES AM, 2019).

Pacientes em situação de vulnerabilidade social, como os moradores de rua, dispõe de grande dificuldade de acompanhamento do tratamento para tuberculose e consequentemente acabam realizando 0 abandono do mesmo, pois bloqueia a realização de visita domiciliar, não possibilita ao paciente priorizar seu tratamento garantindo boa alimentação, repouso, uso correto das medicações e acompanhamento multidisciplinar (HINO P, et al., 2018).

REAS/EJCH | Vol.Sup.n.59 | e4263 | DOI: https://doi.org/10.25248/reas.e4263.2020 Página 6 de 9 
Segundo Harter J, et al. (2016), evidenciou que pacientes registrados como moradores de rua, albergados ou apenados foram associados significativamente com o desfecho abandono, definindo essa variável como preditora no abandono do tratamento. Os fatores mais comuns associados as dificuldades dos pacientes para o enfrentamento, controle e prevenção do abandono do tratamento de tuberculose são: o grande período de execução do tratamento, aspectos socioeconômicos, uso de álcool e outras drogas e falta de apoio familiar (HARTER J, et al., 2016).

Dados do ministério da saúde apontam que a cada 10 pessoas que iniciam o tratamento, pelo menos uma abandona os medicamentos. O tratamento dura em média 6 meses, no entanto, é percebível a melhora dos sintomas nas primeiras semanas após o início, já a cura só é garantida ao final do esquema terapêutico (BRASIL, 2002).

O esquema terapêutico incide na administração de medicamentos em doses conjugadas, 4 em 1, sendo, rifampicina, isoniazida, pirazinamida e etambutol, nos primeiros 2 meses, em seguida as doses tornam-se 2 em 1 (rifampicina e isoniazida) (BRASIL, 2002). De acordo com Harter J, et al. (2016), os princípios básicos para o sucesso do tratamento incluem a escolha do esquema terapêutico adequado, em doses corretas e pelo período de tempo recomendado.

Segundo a investigação de Campani STA, et al. (2011), o abandono ao tratamento ocorreu mais frequentemente dentro dos primeiros três meses do tratamento, com a justificativa do longo período de tratamento. A falta de adesão ao tratamento é considerada o maior obstáculo para o controle da doença no campo da saúde pública, apresentando-se como um desafio e contribuindo de modo importante para o surgimento de resistência aos fármacos utilizados (CAMPANI STA, et al., 2011).

Problemas psicossociais como doenças mentais, população sem teto, moradores de rua, etilismo, associação com HIV entre outros, vem sendo relatados como fatores responsáveis em interferir no sucesso ao tratamento (SILVA PF, et al., 2014). No estudo realizado por Campani STA, et al. (2011), houve ligação entre a variável abandono do tratamento com a utilização exorbitante de álcool e drogas. De modo geral, a compulsão por entorpecentes mostrou-se relacionada ao alcoolismo, assomando uma sobreposição de variáveis (SACKSER MA, BORGES AM, 2019).

A família desempenha um papel importante na adesão ao tratamento da doença, pois colabora junto a equipe na supervisão do uso adequado da medicação, garante alimentação regulada e adequada e fortalece o doente emocionalmente durante o período do tratamento (MENDES AM, FENSTERSIFER LM, 2004). Campani STA, et al (2011), afirma que pacientes que não residiam com familiares apresentaram uma taxa de abandono ao tratamento mais significativa.

\section{Papel do profissional enfermeiro na busca ativa e em estratégias de saúde para minimizar o abandono do tratamento de tuberculose pulmonar na atenção primária}

Braga JU, et al. (2012), afirma que a não adesão ao tratamento por pacientes portadores da tuberculose tem sido visto como um dos maiores problemas no controle dessa doença, pois além de danos individuais, isso põe em risco a saúde pública, levando ao insucesso terapêutico e ao desenvolvimento de cepas resistentes.

Paz LNF, et al. (2012), afirma que a ausência do paciente nas consultas agendadas é um indício de que pode haver a intenção de abandono do tratamento. Nessas situações é indispensável a iniciativa imediata da equipe em realizar a busca ativa dos faltosos. Neste sentido, é imprescindível que o profissional enfermeiro saiba reconhecer os sinais de que o paciente pretende realizar o abandono ao tratamento, visto que na maioria das vezes esses sinais podem ser observados em situações do próprio acompanhamento da doença.

Braga JU, et al (2012), avaliou que o preparo dos profissionais em estratégias de comunicação com os pacientes e a família maximiza a adesão ao tratamento. Paz LNF, et al. (2012), complementa que a construção de um plano terapêutico voltado para necessidade de saúde de cada paciente, favorece a formação de vínculo e a abordagem integral, indo além do processo patológico e considerando o doente em seus aspectos culturais, sociais, econômicos e relacionados ao estigma da doença. 
A estratégia fundamental de acompanhamento do paciente é realizada através da visita domiciliar, onde o visitador, seja ele, agente comunitário de saúde, enfermeiro, médico ou assistente social, pode acompanhar mais de perto o desempenho do paciente em relação ao seu tratamento, supervisionando a tomada de medicamentos e esclarecendo as dúvidas que possam existir (JR JBS, 2004). É relevante garantir a comunicação direta da equipe de saúde com a pessoa em tratamento, seja na unidade de saúde, ou no domicilio, otimizando o acompanhamento dos casos de TB (CAMPANI STA, et al., 2011).

O acompanhamento do paciente quando realizado por uma equipe multiprofissional permite o monitoramento da resposta ao tratamento, sendo possível ajustar a dose da medicação e a identificação dos efeitos adversos do fármaco. Sackser MA e Borges AM (2019), defendem que o sucesso terapêutico, o abandono e até a multirresistência dependem muito da informação fornecida pela equipe durante as consultas.

O sucesso no tratamento da tuberculose está intimamente associado a adesão do paciente ao esquema determinado. Nos dias atuais o esquema terapêutico adotado é altamente efetivo e disponibilizado gratuitamente aos usuários do Sistema Único de Saúde (HAHN GV, 2015).

\section{CONSIDERAÇÕES FINAIS}

O enfermeiro exerce um papel fundamental na busca ativa de pacientes que estejam abandonando o tratamento de tuberculose, através da elaboração de estratégias de acompanhamento satisfatórias, favorecendo a adesão ao tratamento, executando políticas públicas já existentes como TDO, encorajando a participação dos pacientes nas consultas de acompanhamento. É imprescindível assegurar o comprometimento dos gestores nas ações intersetoriais, que visem a integralidade da atenção, garantindo a elaboração de uma linha de cuidado. Portanto, conhecer os fatores relacionados ao abandono do tratamento de TB na atenção primária é fundamental para o aperfeiçoamento da estratégia de tratamento.

\section{REFERÊNCIAS}

1. ALENCAR IFPS, et al. Estratégias preventivas da tuberculose na atenção primária à saúde. Revista Eletrônica Acervo Saúde, v. 11, n. 14, p. e1297-e1297, 2019.

2. ARRUDA SMA, et al. Caracterização dos casos de tuberculose notificados em um município prioritário do Brasil, de 2011-2015. Revista Eletrônica Acervo Saúde, v. 11, n. 13, p. e482-e482, 2019.

3. BRAGA JU, et al. Fatores associados ao abandono do tratamento da tuberculose nos serviços de atenção básica em dois municípios brasileiros, Manaus e Fortaleza, 2006 a 2008. Cad Saúde Colet, v. 20, n. 2, p. 225-33, 2012.

4. BRASIL. Implantação do Plano Nacional pelo Fim da Tuberculose como Problema de Saúde Pública no Brasil: primeiros passos rumo ao alcance das metas. Brasil: Boletim epidemiológico, 2018; 11(49): 1-18. Brasília: MS.

5. BRASIL. Ministério da Saúde. Secretaria de Políticas de Saúde. Departamento de Atenção Básica. Manual técnico para o controle da tuberculose: cadernos de atenção básica / Ministério da Saúde, Secretaria de Políticas de Saúde Departamento de Atenção Básica. - 6. ed. rev. e atual. - Brasília: Ministério da Saúde, 2002.

6. CAMPANI STA, et al. Fatores preditores para o abandono do tratamento da tuberculose pulmonar preconizado pelo Ministério da Saúde do Brasil na cidade de Porto Alegre (RS). Jornal Brasileiro de Pneumologia, v. 37, n. 6, p. 776782, 2011.

7. COUTO DS, et al. Fatores determinantes para o abandono do tratamento da tuberculose: representações dos usuários de um hospital público. Saúde em Debate, Rio de Janeiro, v. 38, n. 102, p. 572-581, set. 2014.

8. FACURI CO, et al. Violência sexual: estudo descritivo sobre as vítimas e o atendimento em um serviço universitário de referência no Estado de São Paulo, Brasil. Cadernos de Saúde Pública, v. 29, p. 889-898, 2013.

9. FERREIRA MRL, et al. O vínculo no tratamento da tuberculose na atenção primária à saúde: uma revisão integrativa. Revista Brasileira em Promoção da Saúde, v. 32, 2019.

10. FRAGA IMN, et al. Adesão ao tratamento da tuberculose e a contribuição do enfermeiro. In: Congresso Internacional de Enfermagem. 2019.

11. FREITAS PC, et al. Performance do enfermeiro/equipe de enfermagem na dispensação de materiais para assistência ao usuário no domicílio. Rev. Gaúcha Enferm. vol.41 no.spe Porto Alegre, 2020.

12. HAHN GV. Incluindo o projeto terapêutico singular na agenda da atenção básica em contexto de vulnerabilidade e não adesão ao tratamento da tuberculose. 2015.

13. HARTER J, et al. Avaliação do acompanhamento e desfecho de casos de tuberculose em município do sul do Brasil. Revista Gaúcha de Enfermagem, v. 37, n. 1, 2016.

14. HINO P, et al. Tuberculosis control from the perspective of health professionals working in street clinics. Rev LatinoAm Enfermagem. 2018.

15. JR JBS. Tuberculose: guia de vigilância epidemiológica. J Bras Pneumol 2004.

REAS/EJCH | Vol.Sup.n.59 | e4263 | DOI: https://doi.org/10.25248/reas.e4263.2020 Página 8 de 9 
16. LIMA EA, et al. TUBERCULOSE NA ATENÇÃO PRIMÁRIA À SAÚDE. Mostra Interdisciplinar do curso de Enfermagem, v. 3, n. 1, 2019.

17. MENDES AM, FENSTERSIFER LM. Tuberculose: por que os pacientes abandonam o tratamento? Bol Pneumol Sanit 2004.

18. MS (Ministério da Saúde). Plano Nacional de Controle da Tuberculose, 1999.

19. PAZ LNF, et al. Efetividade do tratamento da tuberculose. Jornal Brasileiro de Pneumologia, v. 38, n. 4, p. 503-510, 2012.

20. SACKSER MA, BORGES AM. Razões que levam os pacientes com tuberculose a abandonarem o tratamento: perspectivas do enfermeiro. Revista Enfermagem Atual InDerme, v. 87, n. 25, 2019.

21. SILVA PF, et al. Fatores associados ao abandono do tratamento da tuberculose pulmonar no Maranhão, Brasil, no período de 2001 a 2010. Cad. Saúde Pública 30 (8) Ago 2014.

22. SOUSA LO, et al. AS DIFERENTES ABORDAGENS DE PESQUISA CIENTÍFICA E SUAS CLASSIFICAÇÕES. In: 2 ำ Workshop de Inovação, Pesquisa, Ensino e Extensão. 2016.

23. SOUZA MT, et al. Revisão integrativa: o que é e como fazer. Einstein, v. 8, n. 1 Pt 1, p. 102-6, 2010. 